\title{
Scapular spine base fracture with long outside-in superior or posterior screws with reverse shoulder arthroplasty
}

\author{
Osman Nuri Eroğlu ${ }^{1,2}$, Buğra Hüsemoğlu ${ }^{2}$, Onur Başçı ${ }^{1}$, Mustafa Özkan ${ }^{1}$, Hasan Havitçığlu ${ }^{1,2}$ \\ Onur Hapa ${ }^{1}$ \\ ${ }^{1}$ Department of Orthopedics and Traumatology, Dokuz Eylül University School of Medicine, İzmir, Turkey \\ ${ }^{2}$ Department of Biomechanics, Graduate School of Sciences, Dokuz Eylül University, İzmir, Turkey
}

\begin{abstract}
Background: The purpose of the present study was to determine how long superior screws alone or in combination with posterior placement of metaglene screws protruding and penetrating into the scapular spine in reverse total shoulder arthroplasty affect the strength of the scapular spine in a fresh cadaveric scapular model.

Methods: Seven fresh cadaver scapulas were allocated to the control group (short posterior and superior screws) and seven scapulars to the study group (spine base fixation with a four long screws, three with both long superior and long posterior screws).

Results: The failure load was lower in the spine fixation group (long screw, $869 \mathrm{~N}$ vs. short screw, 1,123 N); however, this difference did not reach statistical significance ( $\mathrm{p}>0.05$ ). All outside-in long superior or superior plus posterior screws failed due to scapular spine base fracture; failures in the short screw group were due to acromion fracture. An additional posterior outside-in screw failed to significantly decrease the failure load of the acromion spine.

Conclusions: The present study highlights the significance of preventing a cortical breach or an outside-in configuration when a superior or posterior screw is inserted into the scapular spine base.
\end{abstract}

Keywords: Reverse shoulder arthroplasty; Biomechanical testing; Acromion fracture

\section{INTRODUCTION}

Scapular spine fracture is a serious complication of reverse total shoulder arthroplasty (RTSA) and often is caused by trauma or of insidious onset with no explicit trauma. The incidence of scapular fractures occurring after RTSA was reported to be between $1.3 \%$ and $10.2 \%$ [1-4]. In a particular study, 400 RTSA patients were analyzed and three discrete scapular fracture patterns were described: avulsion fractures of the anterior acromion (type I), fractures of the acromion posterior to the acromioclavicular joint (type II), and fractures of the scapular spine (type III) [1].

The mean time from diagnosis to surgery can be up to 9 months (range, 1.3-24 months). Regardless of treatment, both conservative and surgery patients reported inferior function after fracture compared with initially after RSA [2,4-6]. Osteoporosis increases the risk of fracture [7]. Although it has been attempted

Received: April 6, $2021 \quad$ Revised: April 28, $2021 \quad$ Accepted: May 1, 2021

Correspondence to: Osman Nuri Eroğlu

Department of Orthopedics and Traumatology, Dokuz Eylül University School of Medicine, Inciralti Mahallesi Mithatpaşa Cad. no. 1606, Balçova, Izmir, Turkey

Tel: +90-50-6506-7156, Fax: +90-23-2412-3379, E-mail: onurieroglu@gmail.com, ORCID: https://orcid.org/0000-0002-9807-239X

Financial support: None.

Conflict of interest: None.

Copyright@ 2021 Korean Shoulder and Elbow Society.

This is an Open Access article distributed under the terms of the Creative Commons Attribution Non-Commercial License (http://creativecommons.org/licenses/by-nc/4.0/) which permits unrestricted non-commercial use, distribution, and reproduction in any medium, provided the original work is properly cited. 
to link surgical technique (baseplate orientation, screw length, and distance from screw tip to edge of bone) or other parameters (e.g., intact rotator cuff or design changes like lateralized humeral stem or glenoid side leading to an increase in deltoid stress) to the etiopathogenesis of postoperative acromion or spine fracture, no such associations have reached statistical significance. However, $41 \%-57.1 \%$ of the fractures occurred in association with the tip of the long superior or posterior screw [2,5,7]. A recent biomechanical study reported a lower failure load with superior plus inferior metaglene screw constructs compared to the only inferior screw group, with a failure mode of scapular fracture rather than spine or acromion fractures seen clinically [8].

There is no study in the literature concerning the effect of baseplate superior or posterior screw fixation of the scapular spine on the occurrence of type 3 (base of scapular spine) fractures. The purpose of the present study was to compare the effects of long superior and/or posterior screws protruding and penetrating into the scapular spine on the strength of the scapular spine in a fresh cadaveric scapular model. The hypothesis was that a long outside-in superior baseplate screw will lead to scapular base fracture, and further long posterior outside-in screw fixation will cause fracture at lower load compared to a shorter screw.

\section{METHODS}

This study was approved by the Institutional Review Board of Dokuz Eylül University.

Fourteen fresh frozen cadaver shoulders were used for this study. The mean age of the cadavers was $68 \pm 8$ years (six female and eight male). There were no signs of fracture or macroscopic damage to any tendons or joint surface. The cadavers were allowed to thaw at room temperature overnight before testing and dissection.

Seven scapulae were allocated to the control group (short posterior and superior screw), while seven scapula were in the study group (four long superior screw+long anterior screw [L1], three long superior+long posterior screw [L2]). All long superior and long posterior screws were inserted to the scapular base, and all long anterior screws were inserted to the coracoid base. At least two long screws were planned to be used in the long screw group. One of these long screws was the superior screw to be inserted from the inside-out and targeting the scapular spine. Further, the other long screw was inserted from the anterior by targeting the coracoid basis through the bone (L1) or from the inside to the outside posteriorly, again targeting the scapula spine (L2). In the L1 group, the focus was to minimize the effect of the screw on spine breakage by sending it to the second long coracoid head from the anterior. All surgeries were performed by the lead author. The same 38-mm glenosphere baseplate (DePuy Synthes, Raynham, MA, USA) was used. The metaglene should ideally be positioned on the lower circular area of the glenoid bone. The metaglene central peg is positioned in the center of the inferior circle of the glenoid. The vertical metaglene marking was aligned with the base of the coracoid process superiorly. A $42 \mathrm{~mm} \times 3.5$ $\mathrm{mm}$ screw was used as the long screw, and the short screw was 22 $\mathrm{mm} \times 3.5 \mathrm{~mm}$. X-ray and gross inspection were performed to verify screw location.

The scapulae were cemented into a wooden block after being positioned with a frame. The scapulae were mounted to a materials testing system (AG-I $10 \mathrm{kN}$; Shimadzu, Kyoto, Japan) with the aid of a customized bone-cement junction conus covering the acromion but without contact with any part of the spine. This approach was used to achieve greater physiological force transmission along the length of the acromion compared to single-point loading (Fig. 1) [9]. A 1-N preload was applied for 10 seconds, and each specimen was loaded at a constant rate of 0.5 $\mathrm{mm} / \mathrm{s}$ until fracture of the acromion or base of the spine. The load $(\mathrm{N})$ versus displacement $(\mathrm{mm})$ was recorded until failure.

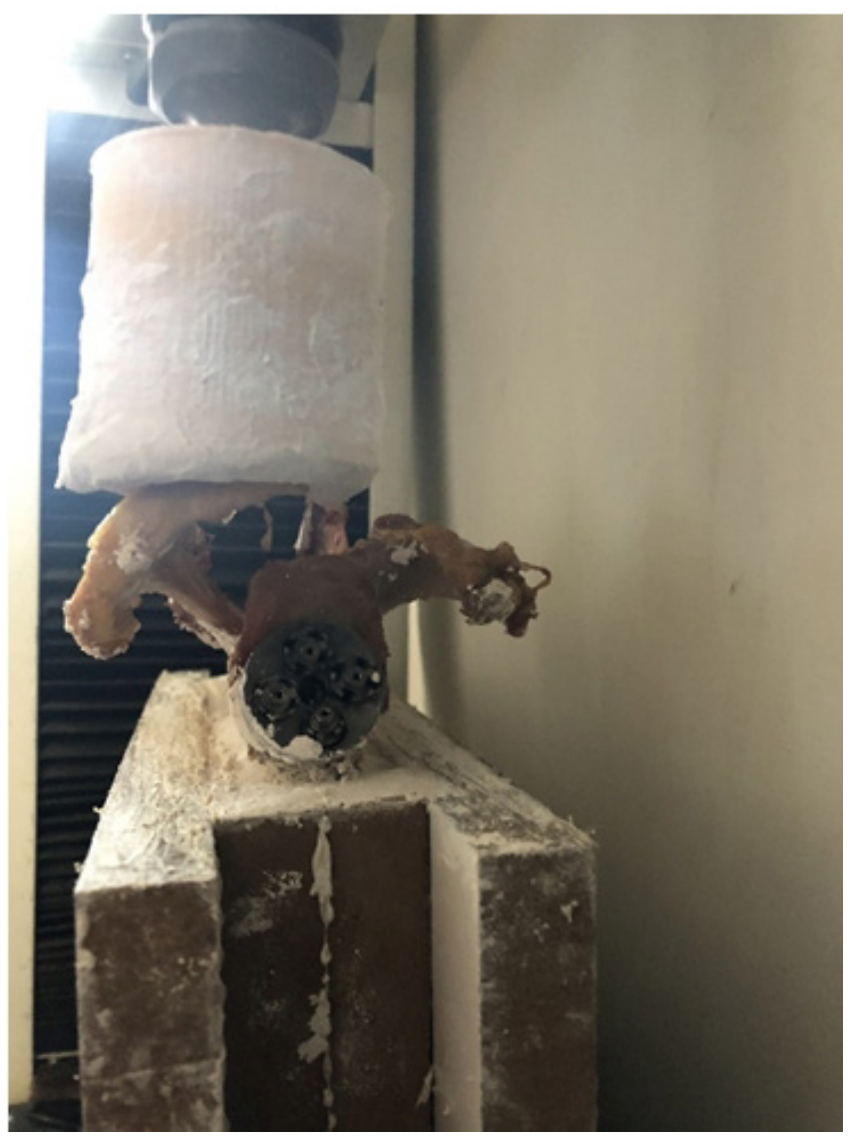

Fig. 1. Test set up. 
The ultimate tensile load was considered the peak force. Stiffness was calculated by determining the slope of the load-displacement curve with the use of a best-fit line on the load versus displacement curve. The ultimate load $(\mathrm{N})$ and stiffness $(\mathrm{N} / \mathrm{mm})$ were measured.

\section{Statistical Analysis}

Data analysis was performed using IBM SPSS ver. 20.0 (IBM SPSS Corp., Armonk, IL, USA). The Mann-Whitney U-test was used to analyze the difference between the mean values of tested variables among the groups. First, the spine fixation group $(n=7)$
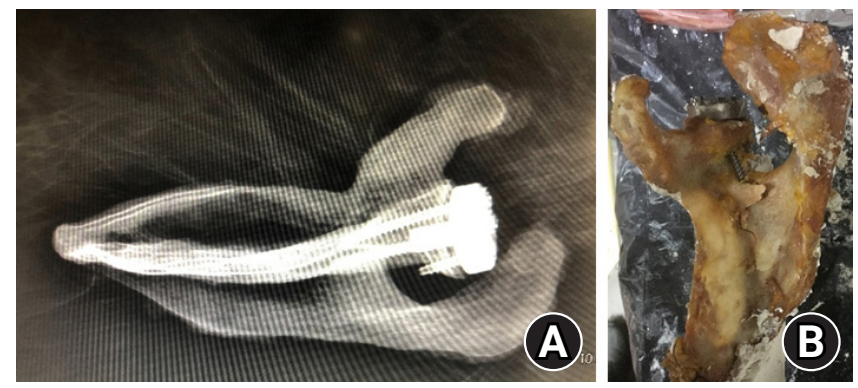

Fig. 2. (A) Long superior and long anterior outside-in posterior screws. (B) Acromion spine base fracture. was compared with the control group $(n=7)$. Then the spine fixation group with a base of spine (type $3, n=6$ ) fracture (Fig. 2) was compared with the control group. A post-hoc power analysis was performed for the spine fracture group in comparison with the control group. A p-value less than 0.05 was considered statistically significant.

\section{RESULTS}

Specimen data are given in Table 1. All specimens in the long screw group except specimen number 7 (long superior and long anterior screws) in the L1 group failed due to acromion spine fracture (type 3) (Fig. 3). In the short screw group, specimen number 7 failed due to acromion fracture (types 1 and 2) (Fig. 4 ). There was no significant difference in mean ultimate load and stiffness when the long screw group was compared to the short screw group $(\mathrm{p}=0.27$, $\mathrm{p}=0.8$ ). This was similar when specimen number 7 at L1 was excluded (no outside-in screw, failure type $1 ; n=6$ ) and compared to the short screw group ( $p=0.25, p=0.77$, respectively).

The mean load and stiffness tended to be lower for L1 compared to L2. Specimen number 13 in the L2 group had superior screw performance without an outside-in configuration (all in-

Table 1. Specimen data

\begin{tabular}{|c|c|c|c|}
\hline Group (specimen no.) & Ultimate load (N) & Stiffness (N/mm) & Fracture type \\
\hline \multicolumn{4}{|c|}{ Long superior and anterior screw $(\mathrm{L} 1, \mathrm{n}=4)$} \\
\hline 1 & 452 & 5 & 3 \\
\hline 2 & 1,090 & 6 & 3 \\
\hline 4 & 789 & 14 & 3 \\
\hline 7 & 1,080 & 23 & 1 \\
\hline Mean \pm SD & $777 \pm 319$ & $8 \pm 4$ & \\
\hline \multicolumn{4}{|c|}{ Long superior and posterior screw $(\mathrm{L} 2, \mathrm{n}=3)$} \\
\hline 10 & 615 & 21 & 3 \\
\hline 12 & 645 & 8 & 3 \\
\hline 13 & 1,413 & 25 & 3 \\
\hline Mean \pm SD & $891 \pm 452$ & $18 \pm 8$ & \\
\hline Total (L1+L2) & $869 \pm 337$ & $14 \pm 8$ & \\
\hline \multicolumn{4}{|l|}{ Short screw $(n=7)$} \\
\hline 3 & 1,098 & 12 & 2 \\
\hline 5 & 1,919 & 13 & 2 \\
\hline 6 & 1,299 & 19 & 1 \\
\hline 8 & 1,736 & 20 & 2 \\
\hline 9 & 335 & 18 & 1 \\
\hline 11 & 891 & 13 & 1 \\
\hline 14 & 767 & 7 & 1 \\
\hline Mean \pm SD & $1,123 \pm 513$ & $15 \pm 4$ & \\
\hline
\end{tabular}

No significant difference for the mean ultimate load and stiffness when the long screw group was compared to the short screw group ( $\mathrm{p}=0.27$, $\mathrm{p}=0.8$. This was similar when specimen number 7 at $\mathrm{L} 1$ was excluded $(\mathrm{n}=6)$ and compared to the short screw group ( $\mathrm{p}=0.25$, $\mathrm{p}=0.77$ respectively). SD: standard devitaion. 
side at the base of spine), while a posterior screw having outside-in fixation into the scapular spine failed with scapular spine base fracture (type 3). A post-hoc power analysis for spine fracture $(n=6)$ comparison to the short screw group $(n=7)$ yielded $0.18(\%)$ for ultimate load and 0.064 (\%) for stiffness.

\section{DISCUSSION}

The main finding of the study was that when the superior metaglene screw (with or without a posterior or anterior screw) enters into the base of the spine with an outside-in configuration, it

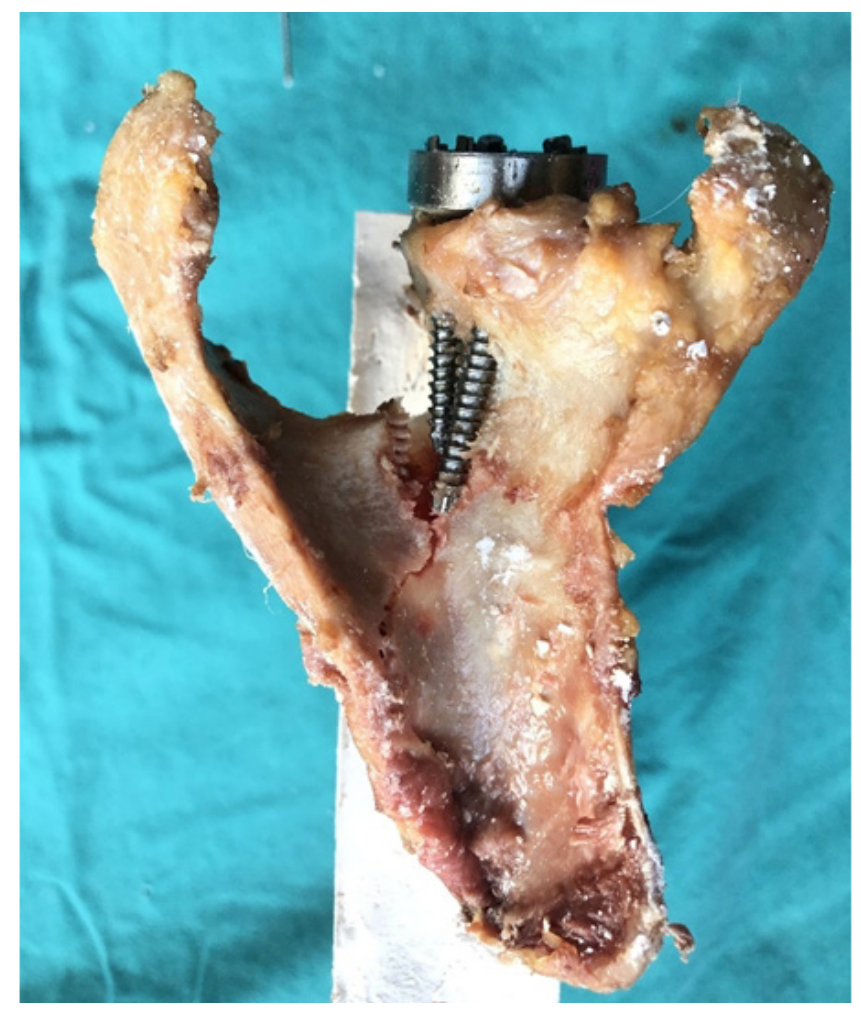

Fig. 3. Two long outside-in screws associated with acromion spine base fracture. leads to a fracture at the base of the scapular spine compared to the short screw "not touching the scapula spine" group, which failed due to acromion fracture. Only one specimen (No. 7 in the L1 group) did not cause a scapula spine base fracture due to fixation of the spine base without an outside-in configuration. However, due to the small number of specimens, the present study failed to show a decrease in ultimate load of the acromion spine when two long screws were used instead of two short screws.

Many clinical studies have highlighted the possible relationship between long superior or posterior screws and later occurrence of scapula spine base fracture $[2,5,7]$. However, due to the small number of occurrences, statistically significant conclusions could not be made [2,5,7]. A recent review also pointed out how scapula spine stress fracture might be related to malposition of the superior 12 o'clock and the posterior (9 o'clock) screws [1]. They advocate that the superior screw be $\leq 24 \mathrm{~mm}$ and angled into the base of the coracoid to prevent nerve injury due to extraosseous placement [10-12]. This was also true for the posterior screw. They advocate avoiding long posterior screws $(\leq 20 \mathrm{~mm})$ to prevent breaching of the spinoglenoid notch and injuring the suprascapular artery and nerve with possible scapular spine fracture.

Whatever treatment is chosen, inferior function is reported after fracture compared with immediately after RSA [2,5,6]. Type 3 fracture (base of acromion spine) especially is associated with painful non-union, which might lead to a greater need for surgical intervention [1]. A recent biomechanical study favored locking the compression plate instead of the lateral clavicular plate or reconstruction plate [9].

There is only one biomechanical study in the literature describing the relationship between superior screws and scapula fractures. Kennon et al. [8] reported that one additional superior screw in addition to the inferior screws led to lower scapula failure load $(1,077 \mathrm{~N}$ vs. $1,970 \mathrm{~N})$, similar to that in our short screw group. They used embalmed cadaveric specimens instead of fresh,
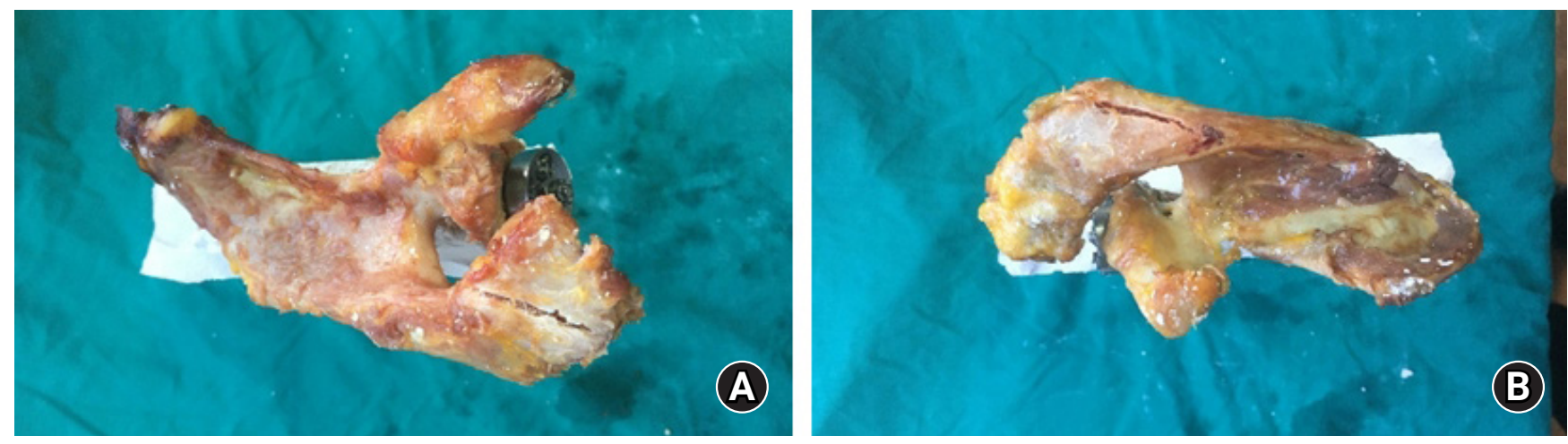

Fig. 4. (A) Type 1 acromion tip fracture (specimen 7). (B) Type 2 acromion fracture (specimen 8). 
as were used in the present study. Their test apparatus was direct compression force from the humerus to scapula. The resultant failure mode was scapula fracture instead of acromion or scapula spine-based fracture, which is the type seen clinically, differing from the present study. The metaglene used in that study was unique in that it had six holes allowing three screws to be placed below the central cage. However, most implants on the market use four screws, one to be placed below the central cage [8].

Some limitations exist for this study; for example, the force applied on the superolateral aspect of the acromion does not fully reflect the distribution of forces acting on the acromion during active shoulder mobilization after RTSA. Further, the majority of scapula and acromion fractures after reverse arthroplasty is stress-related and atraumatic. However, we fractured the acromion or base of spine and correlated the base fracture to the long outside-in screw testing. Additionally, the testing protocol used in the present study was verified in a previous study, and it mimics physiological deltoid muscle pull on the acromion [9].

Second, this is a biomechanical study aimed to test the strength of a construct at time zero and not the healing response over time. Although there was no macroscopic evidence of damage to any component of the joint, there is a possibility that bone quality differences exist between the specimens tested. Additional bone mineral testing would add useful information regarding interpretation of the results. The supply of fresh frozen human cadavers is limited, and the number of specimens in each group was low $(n=7)$. This low sampling rate prevents conclusion with certainty that the long posterior screws decrease the strength of spine and/or lead to fracture at lower loads. However, other biomechanical studies have utilized six specimens per group [13-16]. Specimen number 13 in the L2 (superior screw "all inside," posterior screw outside-in) group failed at higher load than specimen numbers 10 and 12, which had both screws with outside-in fixation into the scapular spine. Third, samples could not be prepared using the two shoulders of one cadaver because the fresh frozen cadavers were separated only in the form of the upper extremities. Furthermore, the bone quality of cadavers was not measured, so the groups were randomly assigned.

The present study indicates the need for additional studies. First, does this outside-in configuration cause spine base fracture using a more physiological test set-up or in a clinical scenario? Second, can all-inside superior long screws aiming toward the scapular spine base be applied clinically and/or prevent fracture of the scapular spine while increasing fixation strength of the baseplate? Third, does an additional outside-in posterior screw further decrease spine fracture load?

Outside-in long superior screw fixation of the base of the scap-

ular spine led to a base fracture compared to the short screw group, which failed due to acromion fracture. The present study points out the significance of prevention of cortical breach or outside-in configuration when the scapular spine base is fixed with superior or posterior screws.

\section{ORCID}

Osman Nuri Eroğlu

Buğra Hüsemoğlu

Onur Başçı

Mustafa Özkan

Hasan Havitçığlu

Onur Hapa

\section{REFERENCES}

1. Crosby LA, Hamilton A, Twiss T. Scapula fractures after reverse total shoulder arthroplasty: classification and treatment. Clin Orthop Relat Res 2011;469:2544-9.

2. Neyton L, Erickson J, Ascione F, Bugelli G, Lunini E, Walch G. Grammont Award 2018: scapular fractures in reverse shoulder arthroplasty (Grammont style): prevalence, functional, and radiographic results with minimum 5-year follow-up. J Shoulder Elbow Surg 2019;28:260-7.

3. Stevens CG, Murphy MR, Stevens TD, Bryant TL, Wright TW. Bilateral scapular fractures after reverse shoulder arthroplasties. J Shoulder Elbow Surg 2015;24:e50-5.

4. Hess F, Zettl R, Smolen D, Knoth C. Anatomical reconstruction to treat acromion fractures following reverse shoulder arthroplasty. Int Orthop 2018;42:875-81.

5. Ascione F, Kilian CM, Laughlin MS, Bugelli G, Domos P, Neyton L, et al. Increased scapular spine fractures after reverse shoulder arthroplasty with a humeral onlay short stem: an analysis of 485 consecutive cases. J Shoulder Elbow Surg 2018;27: 2183-90.

6. Patterson DC, Chi D, Parsons BO, Cagle PJ Jr. Acromial spine fracture after reverse total shoulder arthroplasty: a systematic review. J Shoulder Elbow Surg 2019;28:792-801.

7. Otto RJ, Virani NA, Levy JC, Nigro PT, Cuff DJ, Frankle MA. Scapular fractures after reverse shoulder arthroplasty: evaluation of risk factors and the reliability of a proposed classification. J Shoulder Elbow Surg 2013;22:1514-21.

8. Kennon JC, Lu C, McGee-Lawrence ME, Crosby LA. Scapula fracture incidence in reverse total shoulder arthroplasty using screws above or below metaglene central cage: clinical and biomechanical outcomes. J Shoulder Elbow Surg 2017;26:1023-30. 
9. Kicinski M, Puskas GJ, Zdravkovic V, Jost B. Osteosynthesis of type III acromial fractures with locking compression plate, lateral clavicular plate, and reconstruction plate: a biomechanical analysis of load to failure and strain distribution. J Shoulder Elbow Surg 2018;27:2093-8.

10. DiStefano JG, Park AY, Nguyen TQ, Diederichs G, Buckley JM, Montgomery WH 3rd. Optimal screw placement for base plate fixation in reverse total shoulder arthroplasty. J Shoulder Elbow Surg 2011;20:467-76.

11. Mayne IP, Bell SN, Wright W, Coghlan JA. Acromial and scapular spine fractures after reverse total shoulder arthroplasty. Shoulder Elbow 2016;8:90-100.

12. Molony DC, Cassar Gheiti AJ, Kennedy J, Green C, Schepens A, Mullett HJ. A cadaveric model for suprascapular nerve injury during glenoid component screw insertion in reverse-geometry shoulder arthroplasty. J Shoulder Elbow Surg 2011;20:1323-7.
13. Ahmed M, Young BT, Bledsoe G, Cutuk A, Kaar SG. Biomechanical comparison of long head of biceps tenodesis with in terference screw and biceps sling soft tissue techniques. Arthroscopy 2013;29:1157-63.

14. Arvesen JE, Gill SW, Sinatra PM, Eng M, Bledsoe G, Kaar SG. Biomechanical contribution of tension-reducing rotator cuff sutures in 3-part proximal humerus fractures. J Orthop Trauma 2016;30:e262-6.

15. James J, Allison MA, Werner FW, McBride DE, Basu NN, Sutton LG, et al. Reverse shoulder arthroplasty glenoid fixation: is there a benefit in using four instead of two screws. J Shoulder Elbow Surg 2013;22:1030-6.

16. Pastor MF, Kraemer M, Hurschler C, Claassen L, Wellmann M, Smith T. Transfer of the long head of biceps to the conjoint tendon: a biomechanical study. Clin Biomech (Bristol, Avon) 2016;32:80-4. 